\title{
Design of Low-Profile Compliant Transmission Mechanisms
}

\author{
Frederic H. Giraud, Zhenishbek Zhakypov, and Jamie Paik*, Member, IEEE
}

\begin{abstract}
Robotic origami design allows creating meso-scale robotic systems and mechanisms not limited by degrees of freedom, miniaturization and assembly downsides of conventional transmission mechanisms. However, unlike the traditional rigid approaches, robotic origami application has been limited by the complex deformation and kinematics of the compliant joints and actuation based on active materials or conventional electric motors. To generalize their application at meso-scale requires a combination of the predictability of traditional rigid kinematics and the manufacturing flexibility of robotic origami. Here we present a study of conventional transmission mechanisms, including a slider-crank and cam-follower, made in quasi-2D form by selective machining and stacking of multiple layers of composite material with minimal assembly. Owing to a compliant design powered by low-profile piezoelectric motors, our $5.3 \mathrm{~mm}$ thick and lightweight mechanisms transmit rotational motion to translational movements in and out-ofplane. We develop analytic models that we validate in terms of force and motion output on our prototypes.
\end{abstract}

\section{INTRODUCTION}

Conventional mechanisms composed of 3D rigid bodies, such as slider-cranks, cams, gears, or various arrangements of linkages, have long been used for transmitting or converting forces and motions. These existing transmission mechanisms have proved to be efficient and reliable for achieving desired motions, forces and speeds for specific tasks in macro-scale robotics and machine design. However, their application at the meso-scale, with features of a few hundred micrometers to a few centimeters has been limited due to the difficulty of physical miniaturization, manufacturing and assembly of numerous tiny joints and links that require high-precision machining and a laborious assembly processes. Researchers have also developed various mechanisms at the micro-scale, known as microelectromechanical systems (MEMS), with features ranging from nanometers to micrometers, by replacing the rigid kinematic joints with compliant joints. This allows utilizing different material properties and layer-by-layer manufacturing processes to build active mechanisms without manual assembly. However, the resulting mechanisms generate extremely low forces and reduced motion range, making it difficult to scale up for targeting macro- and mesoscale mechanisms design. Moreover, the cleanroom manufacturing processes based on photolithographic techniques require masks that hinder flexibility and customizability of mechanism design. Developing of meso-scale transmission mechanism combining the customizability of macro-scale mechanisms and rapid manufacturing processes of MEMS is therefore necessary.

Recently, robotic origamis, also known as Robogamis [1]-[3] have become an effective and versatile platform for creating meso-scale robots and machines with fold-

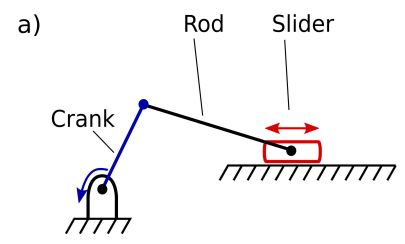

b) Cam Ring follower Slider

c)
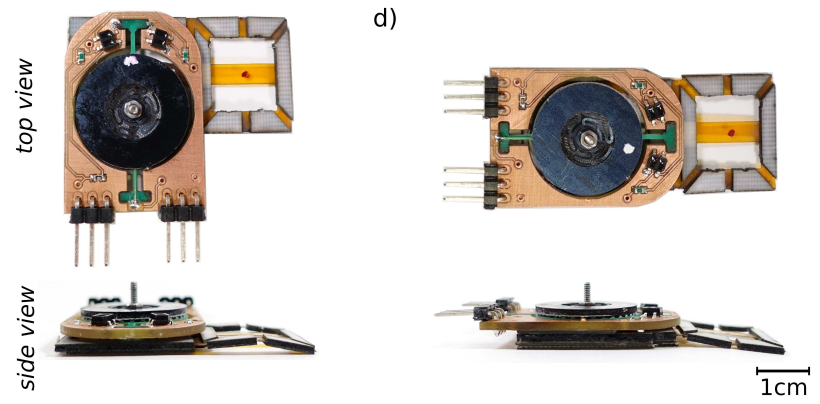

Fig. 1. Transfer of conventional mechanisms into quasi-2D: a) and b) are the schematic views of slider-crank and cam-follower mechanisms. c) and d) represent their multi-layers, low-profile and compliant versions.

able flexure joints, planar linkages, actuators and sensors. They allow rapid fabrication of complete robotic systems from low-profile multi-material functional layers that are laminated layer-by-layer and pop-up folded with minimal manual assembly; known as Smart Composite Microstructures (SCM) [4]. This methodology combines the advantages of traditional mechanism design and MEMS fabrication with multi degrees-of-freedom (DoF) origami movements, enabling high customizability and mass production. Diverse origami robots have been constructed as supports or active structures by distributing bending [5]-[7] or linear [8]-[10] smart actuators in a pattern of one-DoF folding joints [11][14]. As these robots employ direct-drive approach, the actuator design limits their force and motion. Smart materialbased actuators, such as shape memory alloy, generate high forces at lightweight. However, they suffer from high power consumption, low actuation bandwidth $(<1 \mathrm{~Hz})$ and irreversible motion. Pneumatically-driven origami mechanisms overcome some of these challenges due to single actuation [15]-[17] that lacks control over the folding sequence or actuation distributed [18], [19] to fold multi-hinged patterns with complex deformation physics. Use has also been made of origami-based transmission mechanisms actuated by conventional rotary motors using tendons to transmit rotational motion to fold the origami joints [20], [21], or actuate legged robots [22]-[24]. This grants the reproduction of macro-scale mechanisms at small scale with controllable and reversible 
motions. However, such a hybrid approach still requires a 2D to 3D manual assembly of transmission elements, bulky actuators and specific coupling components, which are machined or 3D-printed separately. None of the solutions provide a compact transmission mechanism with tunable and controllable motion and force outputs, and fabricated with minimal assembly effort.

This article presents novel low-profile quasi-2D transmissions with comparable functionality to conventional linkjoint mechanisms for tunable actuation of robotic origami folds. We introduce new slider-crank and cam-follower mechanisms created from a multi-layer lamination process using flexible composite materials. The resulting systems convert rotational DoF to translational movements with high compliance, allowing transmission in- and out-of-plane impossible to achieve with conventional rigid mechanisms. We discuss the capability of combining the proposed scalable mechanisms into a multi-mechanism stack to embed easily diverse transmission systems with reduced numbers of actuators. We also propose a new actuation approach based on planar rotational piezoeletric motors that enables reversible, controllable and locking motions and easy integration. We illustrate the mechanism prototypes fabricated entirely in quasi-2D and assembled to 3D and their embedding into a complex 3-DoF under-actuated origami platform. We model each mechanism analytically and validate the models at noload and loaded conditions with characterization tests. Our work addresses the challenges of mechanical transmission, actuation design, miniaturization and the versatility of mesoscale robots and mechanisms.

The main contributions of this work are:

- Development of new planar and compliant transmission mechanisms and actuation design for meso-scale foldable robots and machines;

- Design and modelling of quasi-2D flat slider-crank and cam-follower with in- and out-of-plane motions;

- Experimental validation of the proposed models with prototypes at unloaded and loaded conditions.

\section{Conventional Transmissions}

Numerous transmission systems are present in the literature and have been used for centuries in conventional mechanics. The field of robotics make important use of rotary motors, which have a well-defined behavior and interesting features in terms of force, speed and integration. Hence, transmissions converting rotation of these actuators find a purpose in numerous mechanical systems and applications. This article focuses on the implementation of recognized and widely used transmission systems converting rotation into translation: a slider-crank and a cam-follower. This section gives an overview of their characteristics.

\section{A. Slider-crank}

Figure 1 a) shows the schematic of a traditional slidercrank mechanism with three main parts: crank, rod and slider. When a rotatory actuator drives the crank, the rod attached to it moves, leading to slider translation. Because the force applied to the slider is not horizontal, its displacement is usually constrained by a slot. In order to ensure a complete rotation of the crank, the Grashof's condition regarding the choice of the links' length has to be satisfied.

With the parameters of Fig. 2 a), the equation that links the displacement $x$ to the crank rotation angle $\theta$ can be found in [25] is,

$$
x=a \cdot \cos (\theta)+b \cdot \cos (\mu)
$$

with a transmission angle $\mu$ linked to $\theta$ by

$$
\mu=\arcsin \left(\frac{a \cdot \sin (\theta)-c}{b}\right)
$$

with, a the crank size, $b$ the mod size and $c$ the offset between the rotor's center and the slider displacement axis.

a)
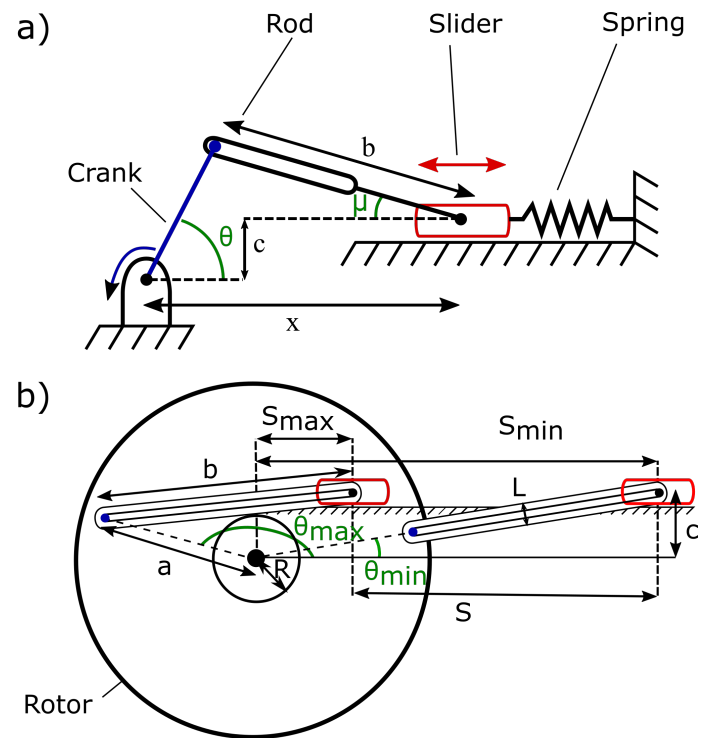

C)

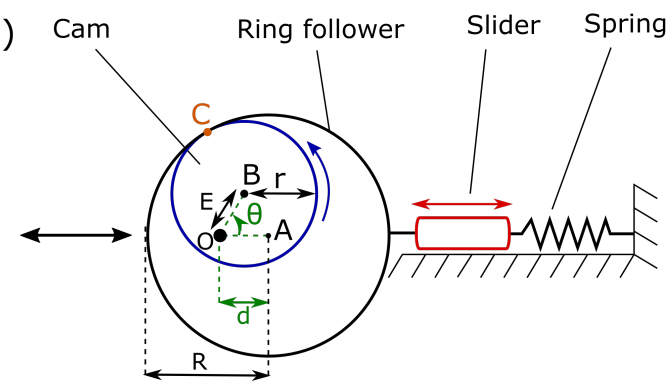

Fig. 2. A low-profile mechanism schematic used to build kinematic models a) Offset slider-crank b) Represents the slider-crank mechanism's limitation in terms of position. $\theta_{\max }$ is reached when the rod is in contact with the spacer and determines the maximal follower position $s_{\max } . \theta_{\min }$ is reached when rod and crank are parallel, which leads to the follower's minimal position $s_{\min }$; c) Cam-follower

\section{B. Cam-follower}

A cam-follower mechanism transforms a rotation into a linear translation and uses the shape of the cam and follower to tune the output motion, force and speed. The follower choice defines the design method for the cam's shape.

While several types of followers exist, the most common are simple roller or flat-surface followers. Their displacement 
is usually constrained to a translation by a slot, but they can also have one end attached to a pivot in the case of oscillating followers. The contact with the cam can be made at one or multiple points and should avoid friction for optimal force transmission to prevent mechanical wear.

The cam itself can vary from a simple eccentric circle to a complex non-uniform shape. Their design starts by choosing the follower motion profile adapted to the application speed and acceleration, such as a simple harmonic motion for example. Then, the follower type defines the method to find the outline's parametric equations. Several optimization methods to minimize the cam's surface, or smooth its curves to improve high acceleration performance, can be found in the literature [26].

However, all the existing work regarding slider-crank and cam-follower transmission cannot be directly translated to the $2 \mathrm{D}$ plane.

\section{DESIGN AND MODELling OF MULTI-LAYERS LOW-PROFILE TRANSMISSIONS}

\section{A. General design considerations}

This paper aims at designing low-profile transmissions by adding compliance to conventional rigid systems, in order to transmit planar motions to out-of-plane configurations. Therefore, their design requires selective arrangement of multi-material layers with variable compliance. This section focuses on the slider-crank and cam-follower transfer to this low-profile, multi-layers compliant mechanism. Even if these transmissions working principles differ, they both aim to translate a slider connected to a load. To ensure movements in- and out- of plane, the slider linked to the core mechanism has to be flexible. Consequently, the mechanism designs requires layers made of flexible material that are used as joints between structural links, and also compose the rod, slider and follower of the cam to provide compliance and allow the overall structure to fold.

However, two constraints arise from the use of flexible layers:

- First, they are more fragile than rigid ones, affecting the overall solidity of the mechanism. To address this issue, we stacked flexible layers to create components with tuned robustness and stiffness, depending on the functionality.

- Second, the mechanism can not push the flexible slider, which will buckle and be unable to move the follower. Consequently, we designed our mechanisms to be uncoupled from the rotor during this phase and added a spring to pull back the follower to its initial resting state. In our case, the spring consists of an integrated origami water-bomb pattern. Nonetheless, it could be designed, or chosen externally from the mechanism, depending on the needs.

We used rigid materials for the structure's skeleton to increase its robustness, where the transmissions' flexibility is not affected. a)

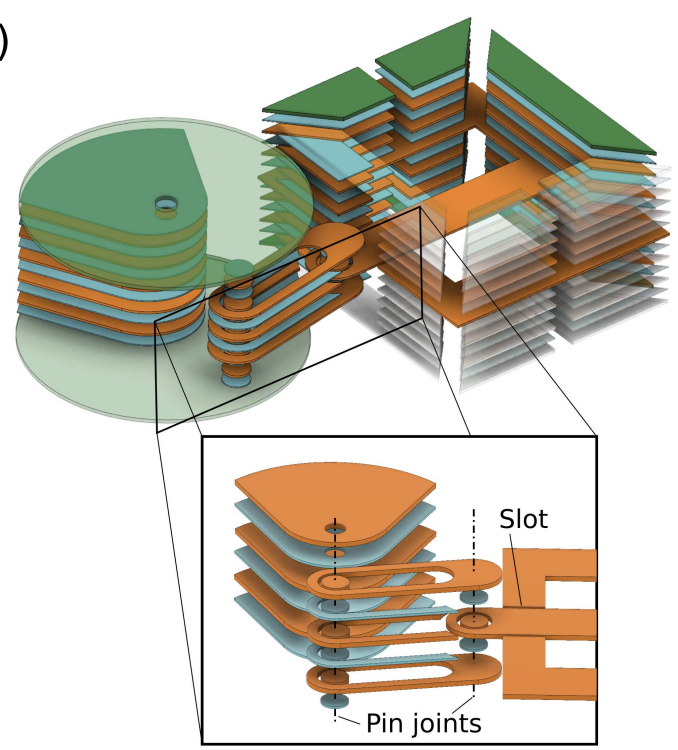

b)

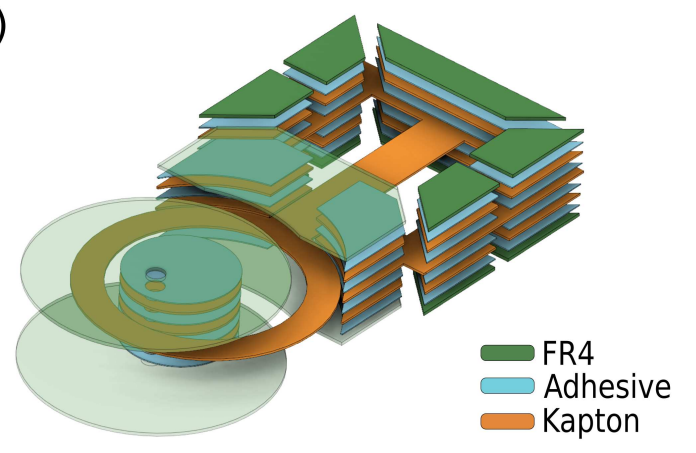

Fig. 3. Layer-by-layer representation of a) Slider-crank and b) Camfollower mechanisms

\section{B. Slider-crank}

1) Design: The developed low-profile offset slider-crank mechanism is shown in expanded view in Fig. 3 a) and a picture of the prototype in Fig. $1 \mathrm{c}$ ). As for a conventional slider-crank, this system has a rod, two pivot joints and a slider. The crank is replaced by a rotor of larger diameter including a pin connected to the rod. When activated, the rotor will drive this pin, pulling the rod and hence translating the slider. In order to decouple translation and rotor rotation during the pushing phase, a slot within the rod allows the rotor pin to slide without applying forces on the slider. Therefore, the contact between the rotor's pin and the end of the rod determines the mechanism's phase. In the forward phase, the follower is led by the rotor, while in the other phase, the follower and rod motion are driven by the flexure hinges' spring. As in conventional mechanisms, the slider's motion in constrained to be linear due to the slot created in the internal layers. A gap made within the rotor, allows the rod to slide inside it when the mechanism is active. The gap is formed from two stacks of layers; one with a circular shape acting as a pin connected to the rod and the other having an area large enough to support the structure, while 
allowing maximum displacement of the slider into the rotor.

The general design is composed of layers of different functionality and layout. The two outer layers in green in Fig. 3 a) are rigid and define the mechanism's overall structure. They also provide a solid surface for the piezo-motor to interact with. The inside layers in orange are functional and serve either as spacers to create gaps to minimize friction, or as mechanical elements such as a rod, pin or slider. Adhesive, represented in blue in Fig 3 a) is placed between the functional layers and has a design adapted to its location in the stack to prevent moving parts being glued to structural elements during the manufacturing process.

2) Model: This section presents the equations used to design a slider-crank with stroke, motion and dimensions adapted to a targeted application. Well-known conventional slider-crank kinematic equations given in the previous section are still verified in this low-profile design. Nonetheless, the rotor's spacer adds a limitation in terms of maximal stroke. In this section, we conduct an analysis of this constraint to determine the maximal displacement of the mechanisms. The schematics used to build this model are represented in Figs. 2 a) and b).

As illustrated in Fig. 3 a), the rotor has a gap inside that allows the crank and slider to move. This space is created by spacers with a radius $\mathrm{R}$, placed in the rotor's center and preventing the rod and slider going through the spacer area. Therefore, Grashof's condition setting the requirement to ensure full rotation of a four-bar linkage system cannot be satisfied. Because of this limitation, the maximum rotation angle is set by one of these two independent conditions: 1) Transmission angle $\mu$ (Fig. 2 a) or 2) Spacer radius R (Fig. 2 b).

1) Transmission angle $\mu$ :

The force component on the slider axis should never reach 0 , which means that the transmission angle $\mu$ has to satisfy:

$$
-\frac{\pi}{2}<\mu<\frac{\pi}{2}
$$

From a geometric consideration, the upper boundary condition is satisfied if,

$$
b>a-c
$$

$\mu$ is given by (2) and therefore (3) becomes:

$$
\arcsin \left(\frac{-b+c}{a}\right)<\theta<\pi-\arcsin \left(\frac{-b+c}{a}\right)
$$

which gives us the boundaries of $\theta$ for a chosen $a, b$ and c.

2) Spacer radius $R$ :

As illustrated in Figure $2 \mathrm{~b}$ ), the slider's maximal position is limited by the rod's width L and the spacer's radius $\mathrm{R}$. Assuming that $(0,0)$ are the rotor's center coordinates, the line passing through the two ends of the rod has this equation:

$$
\begin{gathered}
(a \cdot \sin (\theta)-c) \cdot x-(b \cdot \cos (\mu)) \cdot y+a \cdot \cos (\theta) \cdot c \\
-a \cdot \sin (\theta) \cdot[a \cdot \cos (\theta)+b \cdot \cos (\mu)])=0
\end{gathered}
$$

$\mathrm{D}$ is given by the line to point distance relation:

$$
\begin{array}{r}
D=(|a \cdot \cos (\theta) \cdot c-a \cdot \sin (\theta) \cdot[a \cdot \cos (\theta)+b \cdot \cos (\mu)]|) \\
/\left(\sqrt{(a \cdot \sin (\theta)-c)^{2}+(b \cdot \cos (\mu))^{2}}\right)
\end{array}
$$

After simplification, (7) becomes,

$$
D=|a \cdot \sin (\mu+\theta)|
$$

From Fig.2 b), the maximal position is given when,

$$
D=R+\frac{L}{2}
$$

By equating (8) and (9), $\theta_{\max }$ can be determined given all the other parameters are defined by the design.

The final maximal position that can be reached is the smallest $\theta_{\max }$ between 1) and 2).

Regarding the minimal position $s_{\text {min }}$; it is reached when the rod and crank are parallel as shown in $2 \mathrm{~b}$ ). The relation between $s_{\min }$ and $\theta_{\min }$ is found using simple trigonometry:

$$
(a+b) \cdot \cos \left(\theta_{\min }\right)=s_{\min }
$$

The stroke of this mechanism is dependant on $\theta$ limitations and is the distance $s$ between the maximum displacement $s_{\max }$ for $\theta_{\max }$ and the low position $s_{\min }$. Therefore the stroke $s$, given by the design parameters of this system $a, b$, c, $\mathrm{R}, \mathrm{L}$ is:

$$
\begin{gathered}
\qquad s=s_{\max }-s_{\min } \\
\Rightarrow s=a \cdot \cos \left(\theta_{\max }\right) \\
+b \cdot \cos \left(\arcsin \left(\frac{a \cdot \sin \left(\theta_{\max }\right)-c}{b}\right)\right)-(a+b) \cdot \cos \left(\theta_{\min }\right)
\end{gathered}
$$

\section{Cam-follower}

1) Design: An expanded view and a prototype picture of the low profile cam-follower mechanism developed in this research are illustrated in Figs. $3 \mathrm{~b}$ ) and $1 \mathrm{~d}$ ), respectively. The system's working principle is similar to a conventional cam-follower. When activated, the cam rotation pushes the surface of a ring-shaped follower, pulling a flexible slider linked to the load, leading to its translation. During the return phase, the constant of the chosen spring decides whether or not there is contact between the cam and follower and therefore, whether rotor rotation and follower translation are dependent. A slot in the structural layers constrains the slider's motion to be linear. When activated, slider and follower can move inside the rotor as a result of the a gap created by the layers comprising the cam itself.

The thinness of the inside material and hence flexibility makes the follower have a curved shape. There is indeed an important friction between cam and follower that leads to unwanted tangential forces. In the case where the follower has a flat surface for example, it would be driven to the side, leading to slider torsion and consequently mechanical failure. This design uses a ring as follower, which prevents 
the tangential motion by creating a side contact between the cam and follower during rotation. Hence, although the ring shape increases friction, the slider's displacement is kept linear, ensuring a properly working mechanism. In order to simplify the model, the cam and ring design are two eccentric circles.

Simpler than the slider-crank, the cam-follower needs less functional layers. Slider, flat-follower and cam layer can be part of the same layer or stack of layers. Spacers minimizing friction with the two structural rigid layers are placed on the outside. Adhesive is placed between every layer with a specific design depending on the position in the stack to avoid gluing together moving parts.

2) Model: The mechanism design is shown in figure 2 c) and consists of two eccentric circles. The ring, with a radius $\mathrm{R}$, moves linearly when pushed by the cam of radius r. This section establishes the mathematical equation linking the displacement $\mathrm{d}$ to the cam rotation angle $\theta$.

For every $\theta$, because of our circular design, the ring's center $\mathrm{A}$, cam center $\mathrm{B}$ and contact point $\mathrm{C}$ between cam and ring are aligned. Therefore,

$$
\overrightarrow{A C}=\overrightarrow{A B}+\overrightarrow{B C}
$$

equal to,

$$
\|\overrightarrow{A C}\|=\|\overrightarrow{A B}\|+\|\overrightarrow{B C}\|
$$

Knowing that $\|\overrightarrow{A C}\|=R$ and $\|\overrightarrow{B C}\|=r$ only $\|\overrightarrow{A B}\|$ needs to be found to use 14 .

From Fig. 2 we can see that,

$$
\begin{gathered}
\overrightarrow{A B}=\overrightarrow{A O}+\overrightarrow{O B} \\
\Rightarrow \overrightarrow{A B}=\left[\begin{array}{c}
-d \\
0
\end{array}\right]+\left[\begin{array}{l}
E \cdot \cos (\theta) \\
E \cdot \sin (\theta)
\end{array}\right] \\
\Rightarrow \overrightarrow{A B}=\left[\begin{array}{c}
-d+E \cdot \cos (\theta) \\
E \cdot \sin (\theta)
\end{array}\right]
\end{gathered}
$$

Hence,

$$
\|\overrightarrow{A B}\|=\sqrt{(-d+E \cdot \cos (\theta))^{2}+(E \cdot \sin (\theta))^{2}}
$$

By inserting (18) into (14) we get a second order polynomial equation of unknown $\mathrm{d}$ :

$$
\left.(R-r)^{2}=d^{2}-2 \cdot E \cdot d \cdot \cos (\theta)\right)+E^{2}
$$

having two roots that correspond to the symmetric motion relative to the ring's center:

$$
\begin{aligned}
d(\theta)= & -E \cdot \cos (\theta) \pm \\
& \sqrt{\left(E^{2} \cdot \cos (\theta)^{2}-E^{2}+R^{2}-2 \cdot r \cdot R+r^{2}\right.}
\end{aligned}
$$

One design constraint is given by (20) where $d(\theta)$ exists only if $E^{2} \cdot \cos (\theta)^{2}-E^{2}+R^{2}-2 \cdot r \cdot R+r^{2} \geq 0$. This condition is verified if,

$$
R-r \geq E \cdot \sin \left(\theta_{\max }\right)
$$

with $\theta_{\max }$ the maximum rotation angle for a specific application. Therefore, the condition to satisfy a full rotation of the cam inside the ring is given by,

$$
R-r>E
$$

\section{MANUFACTURING PROCESS OF MULTI-LAYER LOW-PROFILE TRANSMISSION MECHANISMS}

\section{A. Mechanisms}

The systems presented in this paper are created using a layer-by-layer manufacturing process such as the one presented in [7]. It consists of stacking and gluing functional layers of different materials using Polymelt 701 sheets as adhesive and a heat press. For this project, the choice of materials was reduced to Kapton, FR4 and adhesive. $50 \mu \mathrm{m}$ thick Kapton sheets were used because of their flexibility and high mechanical resistance to strain and sandwiched between two $0.3 \mathrm{~mm}$-thick layers of FR4. These materials are temperature resistant and thus suit for heat press.

The manufacturing process is identical for the two mechanisms and presented in Fig. 4. First, the different adhesive, FR4 and Kapton layers are cut by laser, in our case with a Trotec Speedy 400. The layer design has an outline with holes to make manipulation and alignment easier. Problems arise from the Polymelt 701, as it is very thin, making bridges and floating parts prone to breaking and extremely difficult to align when removed from the protective layer. We therefore adjusted the laser parameters to cut though the adhesive without affecting the protective layers, allowing peeling off the useless parts while keeping the ones of interest rigid enough to be manipulated. The resulting adhesives are fixed onto their corresponding FR4 or Kapton layer using a heat press $\left(90 \mathrm{Kg}\right.$ at $110^{\circ} \mathrm{C}$ for $\left.10 \mathrm{~min}\right)$. After a coolingdown period, the protective layers are peeled off, leaving the FR4 or Kapton coated with adhesive. These layers are then aligned and heat pressed using the same parameters to give the final stack. After a second cooling-off period, the resulting layers are placed again into the laser, where outlines and bridges are cut out to give the final mechanism.

Regarding the heat press, a uniform pressure is applied onto the stack of layers. This information needs to be considered in the design phase because of the layers' flexibility, which makes the small central part collapse due to gravity. This would result in a non-flat surface and non-uniform pressure, leading to bad layer adhesion in certain part of the design. For that reason, extra parts, either permanent or temporary, were introduced to support the collapsible parts of the surrounding layers. These support materials are extra pins to support pivots, or bridges designed for places where the device functions would not be affected, aim at putting uniform tension over all the surface and avoiding moving any floating parts. These additional components become useless after manufacture and fall off at the final laser-cutting stage, producing the functional mechanism.

The resulting mechanisms without the actuator have a thickness of $1.25 \mathrm{~mm}$. 


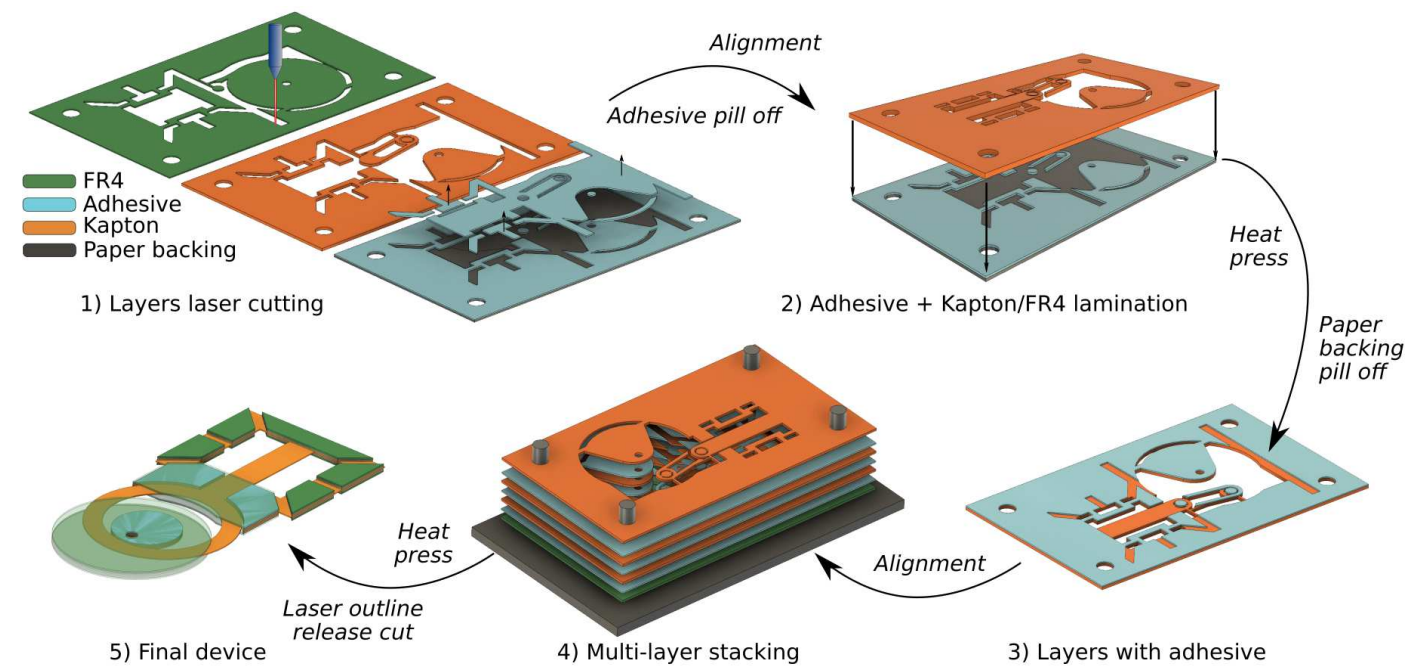

Fig. 4. Manufacturing process used for the mechanisms described in this paper. The different layers are laser cut and corresponding functional and adhesive layers are grouped in pairs. After alignment and heat press, the adhesive is coated onto the functional layers and the protective sheets peeled off. Then, using an alignment platform and heat-press to re-melt the adhesive, all layers are stacked and glue together. Finally, the outline used for the alignment is removed by laser cutting and the resulting mechanism is fully functional.

\section{B. Actuation}

Although our mechanisms are compatible with different types of actuators, such as DC motors, we choose PCBmotors [27], [28] as actuation method. These are made of thin piezo elements with high force density, and can be produced using pick-and-place technique directly on the PCB. Hence, they are easy to integrate into planar devices and thus seem particularly adapted to actuate Robogamis.

The PCBmotors we used have a $20 \mathrm{~mm}$ stator radius in order to minimize the final size of the transmission. With this dimension, the manufacturer ensure a free speed of 1.6 $\mathrm{rev} / \mathrm{s}, 8 \mathrm{Nmm}$ stall torque and a maximum output power of $40 \mathrm{~mW}$.

We fixed the PCBmotors onto the rotor already integrated in the mechanisms, driving them to translate followers. For better integration, we designed a compact PCB embedding motor, sensors and a central bearing to keep the rotation axis in the middle. A second rotor fixed onto the other side of the PCBmotors contains a spring as advised by [28] and teeth, leading to variations in light during the rotation. Consequently, embedded sensors can detect the light modulation and with a comparator and low pass filter, can determine the rotation angle, speed and direction. A spacer is used to mechanically connect the two rotors while ensuring a gap big enough to avoid blocking the motor's rotation. The overall system integrating sensors and actuation in presented by Fig. 5 .

Equipped with PCBmotors, our mechanisms have a thickness of $5.5 \mathrm{~mm}$ for a weight of $5.74 \mathrm{~g}$ for the slider-crank and $6.29 \mathrm{~g}$ for the cam-follower.

\section{EXPERIMENTS AND CHARACTERIZATION}

In order to assess the performance of the mechanisms in terms of motion and force, we ran two experiments at no load and blocked conditions, respectively.

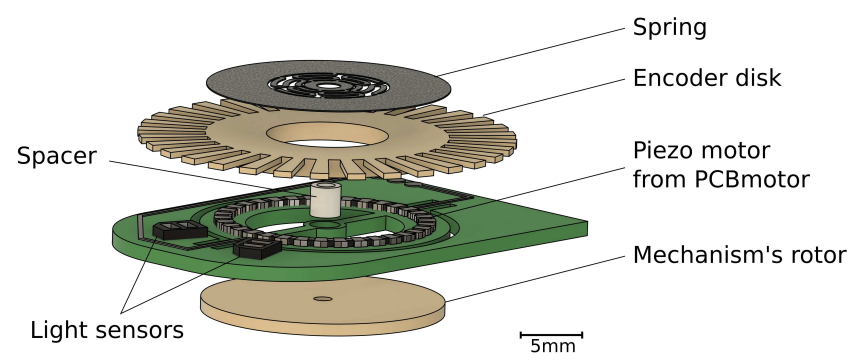

Fig. 5. PCBmotor assembly and sensors integration

\section{A. Kinematics}

This experiment aims to characterize the follower displacement of the mechanisms relative to the motor rotation angle. We use the results to validate the model developed in the design section.

We fixed the two prototypes onto a horizontal surface, unloaded, with a camera positioned above. Each had the follower and rotor equipped with a marker. Once activated, the camera recorded the movement of the whole system and a tracker software extracted the follower displacement and rotor rotation angle. The experimental setup is illustrated in Fig. 6 a).

The motion profiles of the two mechanisms are shown in Fig. 7 a). Only the forward phase is considered because the return phase is uncoupled from the rotor, meaning that there is no relationship between the angle and displacement. Although both mechanisms have an experimental trend similar to the models, the differences between theory and experiment are nonetheless more important for the cam-follower. This can be explained by the flexibility of Kapton, used to make the transmission's internal ring. Indeed, the latter could be deformed by the flexure-hinge's spring force, leading to 
a)

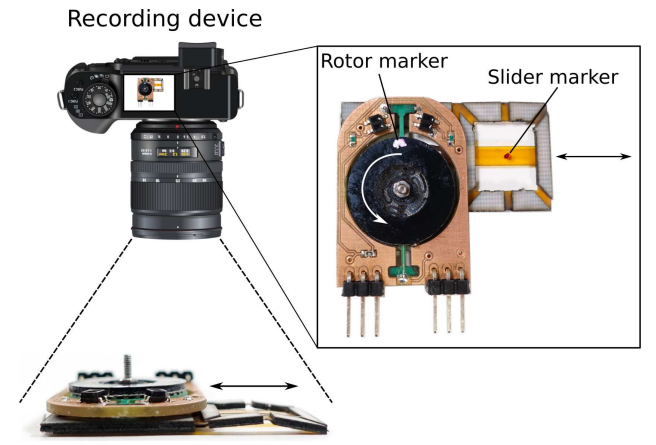

b)

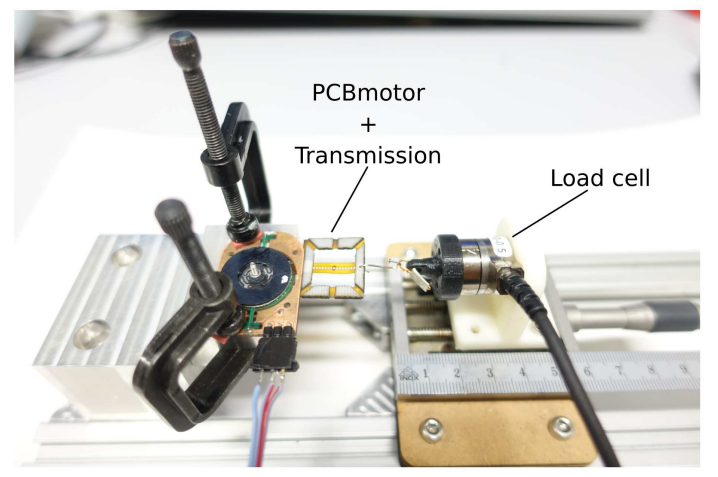

Fig. 6. Experimental setup used to measure the transmissions' force and motion. a) To characterize the motion profile, a camera is used to record the displacement of two markers, placed on the rotor and slider respectively. b) The force of the complete system is measured for different strokes by moving the load cell. Ruler is shown in $\mathrm{cm}$ scale.

the $1 \mathrm{~mm}$ difference in the maximal displacement. Friction between the cam and ring also makes achieving a smooth stroke rise difficult.

\section{B. Pulling force}

We characterized the linear force generated by the $P C B$ motor-actuated slider-crank and cam-follower. A picture of the experimental setup is given in Fig. 6 b). We fixed the prototypes onto a test bench with their followers linked to a load cell. First, we set the transmission to its initial position corresponding to the maximum extension of the follower. After turning on the PCBmotor, we recorded the linear force of the mechanisms in this position. The follower is then moved by steps of $1 \mathrm{~mm}$ for the slider-crank and $0.5 \mathrm{~mm}$ for the cam and the force measured at each step. We conducted this experiment three times to produce a measurement error estimation. The results are shown in Fig. 7 b).

\section{DISCUSSION AND CONCLUSION}

This paper presents the design and models of two planar and compliant transmission mechanisms at meso-scale, a slider-crank and cam-follower. Built using multiple layers of flexible and rigid composite materials, the presented actuatorlink-joint mechanisms provide novel transmission solutions for self-folding robotic origami structures. We also proposed a new compact actuation method, based on flat piezoelectric motors that enable bidirectional rotation with high torque and locking. We described working principles and proposed a)
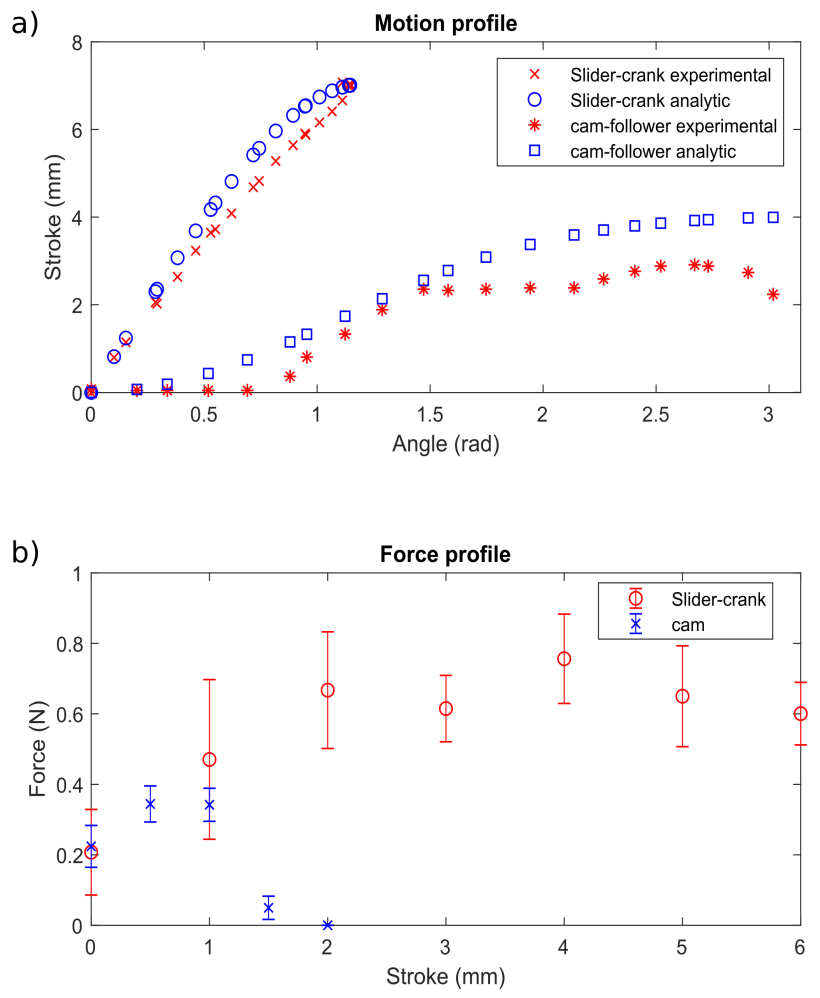

Fig. 7. Experimental results. a) Motion profile: comparison of the stroke, function of the angle, between experimental data and models for unloaded slider-crank and cam-follower transmissions. b) Force profile: measure of the linear force at different strokes for the mechanisms

a generalized design schema for the mechanisms by establishing parameter-based mathematical models to facilitate customized usage. We experimentally characterized the prototype designs and verified their analytic models. The developed slider-crank and cam-follower mechanism designs generate average pulling force of $0.76 \mathrm{~N}$ and $0.35 \mathrm{~N}$ with maximum stroke of $7 \mathrm{~mm}$ and $3 \mathrm{~mm}$, respectively.

Future work requires addressing the several limitations of the proposed mechanisms. Currently, the pin joints lack lubrication to reduce friction losses. The clearances require further optimization to avoid friction and stoppage of the sliding elements to ensure closer behaviour to the mathematical models. The flexible components undergo deformation and are fragile, therefore requiring more investigation into the material properties and geometry. We also aim to implement a design method to adapt various mechanisms in origami, such as four or six bar planar linkages.

In order to demonstrate combination of discrete mechanism layers into more complex multi-mechanism stacks, we constructed a high DoF under-actuated yet compact robotic platform, such as a three DoF origami parallel robot. This system is shown in Fig. 8 and consists of two planar surfaces at the top and bottom, linked by three origami waterbombs on the mechanism's sides. Actuation is achieved by two PCBmotors, each controlling the folding of the two sides independently by changing rotation direction. This device 
has several applications including a single unit as a robotic wrist for a gripper or targeting the same kind of applications than a delta robot. These units could be combined in series to increase their range of motion or in parallel to enhance their force, adapting their features to the targeted application. Future work will aim to model and characterize this platform.

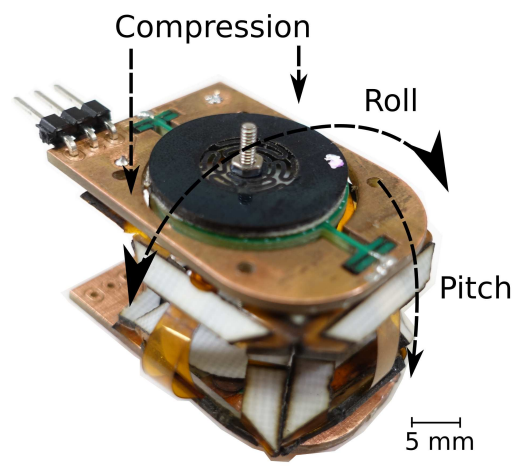

Fig. 8. Three degrees-of-freedom (roll, pitch and compression) underactuated parallel platform.

\section{REFERENCES}

[1] A. Firouzeh and J. Paik, "Robogami: A fully integrated low-profile robotic origami," Journal of Mechanisms and Robotics, vol. 7, no. 2, p. $021009,2015$.

[2] A. Firouzeh, Y. Sun, H. Lee, and J. Paik, "Sensor and actuator integrated low-profile robotic origami," in 2013 IEEE/RSJ International Conference on Intelligent Robots and Systems. Ieee, 2013, pp. 49374944.

[3] J. Paik, "Soft robot design methodology for 'pushbutton'manufacturing," Nature Reviews Materials, vol. 3, no. 6, p. 81, 2018.

[4] R. Wood, S. Avadhanula, R. Sahai, E. Steltz, and R. Fearing, "Microrobot design using fiber reinforced composites," Journal of Mechanical Design, vol. 130, no. 5, p. 052304, 2008.

[5] J. K. Paik, R. K. Kramer, and R. J. Wood, "Stretchable circuits and sensors for robotic origami," in Intelligent Robots and Systems (IROS), 2011 IEEE/RSJ International Conference on. IEEE, 2011, pp. 414420.

[6] Z. Zhakypov, J.-L. Huang, and J. Paik, "A novel torsional shape memory alloy actuator: Modeling, characterization, and control," IEEE Robotics \& Automation Magazine, vol. 23, no. 3, pp. 65-74, 2016.

[7] Z. Zhakypov and J. Paik, "Design methodology for constructing multimaterial origami robots and machines," IEEE Transactions on Robotics, vol. 34, no. 1, pp. 151-165, 2018.

[8] C. D. Onal, R. J. Wood, and D. Rus, "An origami-inspired approach to worm robots," IEEE/ASME Transactions on Mechatronics, vol. 18, no. 2, pp. 430-438, 2013.

[9] M. Salerno, K. Zhang, A. Menciassi, and J. S. Dai, "A novel 4-dof origami grasper with an sma-actuation system for minimally invasive surgery," IEEE Transactions on Robotics, vol. 32, no. 3, pp. 484-498, 2016.

[10] Z. Zhakypov, M. Falahi, M. Shah, and J. Paik, "The design and control of the multi-modal locomotion origami robot, tribot," in 2015 IEEE/RSJ International Conference on Intelligent Robots and Systems (IROS). IEEE, 2015, pp. 4349-4355.

[11] X. Sun, S. M. Felton, R. Niiyama, R. J. Wood, and S. Kim, "Selffolding and self-actuating robots: a pneumatic approach," in Robotics and Automation (ICRA), 2015 IEEE International Conference on. IEEE, 2015, pp. 3160-3165.

[12] M. T. Tolley, S. M. Felton, S. Miyashita, D. Aukes, D. Rus, and R. J. Wood, "Self-folding origami: shape memory composites activated by uniform heating," Smart Materials and Structures, vol. 23, no. 9, p. 094006, 2014.
[13] S. Miyashita, C. D. Onal, and D. Rus, "Self-pop-up cylindrical structure by global heating," in Intelligent Robots and Systems (IROS), 2013 IEEE/RSJ International Conference on. IEEE, 2013, pp. 40654071.

[14] H. Shigemune, S. Maeda, Y. Hara, N. Hosoya, and S. Hashimoto, "Origami robot: A self-folding paper robot with an electrothermal actuator created by printing," IEEE/ASME Transactions on Mechatronics, vol. 21, no. 6, pp. 2746-2754, 2016.

[15] A. Rafsanjani, Y. Zhang, B. Liu, S. M. Rubinstein, and K. Bertoldi, "Kirigami skins make a simple soft actuator crawl," Science Robotics, vol. 3, no. 15, p. eaar7555, 2018.

[16] S. Li, D. M. Vogt, D. Rus, and R. J. Wood, "Fluid-driven origamiinspired artificial muscles," Proceedings of the National Academy of Sciences, p. 201713450, 2017.

[17] R. V. Martinez, C. R. Fish, X. Chen, and G. M. Whitesides, "Elastomeric origami: programmable paper-elastomer composites as pneumatic actuators," Advanced functional materials, vol. 22, no. 7, pp. 1376-1384, 2012.

[18] R. Niiyama, X. Sun, C. Sung, B. An, D. Rus, and S. Kim, "Pouch motors: Printable soft actuators integrated with computational design," Soft Robotics, vol. 2, no. 2, pp. 59-70, 2015.

[19] Z. Zhakypov, M. Mete, J. Fiorentino, and J. Paik, "Programmable fluidic networks design for robotic origami sequential self-folding," in 2019 2nd IEEE International Conference on Soft Robotics (RoboSoft). IEEE, 2019, pp. 814-820.

[20] A. Firouzeh and J. Paik, "Grasp mode and compliance control of an underactuated origami gripper using adjustable stiffness joints," Ieee/asme Transactions on Mechatronics, vol. 22, no. 5, pp. 21652173, 2017.

[21] D. Jeong and K. Lee, "Design and analysis of an origami-based threefinger manipulator," Robotica, vol. 36, no. 2, pp. 261-274, 2018.

[22] S. G. Faal, F. Chen, W. Tao, M. Agheli, S. Tasdighikalat, and C. D. Onal, "Hierarchical kinematic design of foldable hexapedal locomotion platforms," Journal of Mechanisms and Robotics, vol. 8, no. 1, p. 011005, 2016.

[23] P. Birkmeyer, K. Peterson, and R. S. Fearing, "Dash: A dynamic 16g hexapedal robot," in Intelligent Robots and Systems, 2009. IROS 2009. IEEE/RSJ International Conference on. IEEE, 2009, pp. 2683-2689.

[24] S. Felton, M. Tolley, E. Demaine, D. Rus, and R. Wood, "A method for building self-folding machines," Science, vol. 345, no. 6197, pp. 644-646, 2014.

[25] D. H. Myszka, Machines and mechanisms. Prentice Hall, 2004.

[26] J. Angeles and C. S. López-Cajún, Optimization of cam mechanisms. Springer Science \& Business Media, 2012, vol. 9.

[27] H. Ellesgaard, E. Johansen, T. Olesen, H. Andersen, and M. H. Hansen, "Electro-mechanical wave device," May 25 2010, uS Patent 7,723,900.

[28] "Pcbmotor website," https://pcbmotor.com/. 